\title{
Infiltrating angiolipoma of the thoracic wall: A case report
}

\author{
Ayako Hamano $^{1 *}$, Kazuhiro Suzuki ${ }^{1}$, Tsuyoshi Saito ${ }^{2}$, Ryohei Kuwatsuru ${ }^{1}$, Shiaki Oh $^{3}$, Kenji Suzuki $^{3}$ \\ ${ }^{1}$ Department of Radiology, Faculty of Medicine, Juntendo University, Tokyo, Japan \\ ${ }^{2}$ Department of Human Pathology, Faculty of Medicine, Juntendo University, Tokyo, Japan \\ ${ }^{3}$ Department of General Thoracic Surgery, Faculty of Medicine, Juntendo University, Tokyo, Japan \\ Email: *ahamano@juntendo.ac.jp
}

Received 10 April 2013; revised 11 May 2013; accepted 20 May 2013

Copyright (c) 2013 Ayako Hamano et al. This is an open access article distributed under the Creative Commons Attribution License, which permits unrestricted use, distribution, and reproduction in any medium, provided the original work is properly cited.

\begin{abstract}
We describe a case of infiltrating angiolipoma of the thoracic wall, which is an extremely unusual site for this tumor. Infiltrative angiolipoma is a rare benign soft tissue tumor that commonly affects the extremities and the trunk. This is the first report of infiltrative angiolipoma that affects this site. Magnetic resonance imaging (MRI) revealed a high intensity signal on T2-weighted images and contrast enhanced images showed moderate enhancement effect that mimicked tumors of neurogenic or vascular origin. Angiolipoma should be included in a differential diagnosis of the thoracic wall tumor with $\mathrm{T} 2$ high intensity signal and contrast enhancement that is not typical shape and signal for neurogenic tumor or hemangioma.
\end{abstract}

Keywords: Infiltrating Angiolipoma; Chest Wall Tumor; Computed Tomography; Magnetic Resonance Imaging

\section{INTRODUCTION}

Infiltrating angiolipoma is a benign lipomatous lesion that mainly occurs in soft tissues and frequently presents in the extremities and the trunk [1,2]. Angiolipoma histologically comprises adipocytes and angiomatous proliferation. Although pathologically benign, infiltrative angiolipoma frequently infiltrates surrounding muscles, fascia and subcutaneous tissues, and thus wide excision is recommended $[3,4]$. However, diagnosing this type of tumor before surgical excision is difficult. Here, we describe infiltrative angiolipoma of the thoracic wall, which is an extremely unusual site for this tumor. To our best of knowledge, this is the first report in the English literature of an infiltrative angiolipoma of the thoracic wall.

\footnotetext{
"Corresponding author.
}

\section{CASE REPORT}

A 25-year-old asymptomatic man was referred to our institution for resection of a thoracic wall tumor that was incidentally discovered by chest radiography and computed tomography (CT) at a routine health checkup as a round opacity in the left mid lung field with an incomplete border sign (Figure 1). A history of neoplastic or skin diseases was not evident and all laboratory findings were within normal ranges.

The opacity was not calcified on chest radiography. CT of the chest revealed a well-circumscribed dome-like mass with a diameter of $35 \mathrm{~mm}$ at the subpleural region around the right sixth rib without gross lung or bony invasion (Figure 2(a)). Attenuation on unenhanced CT was

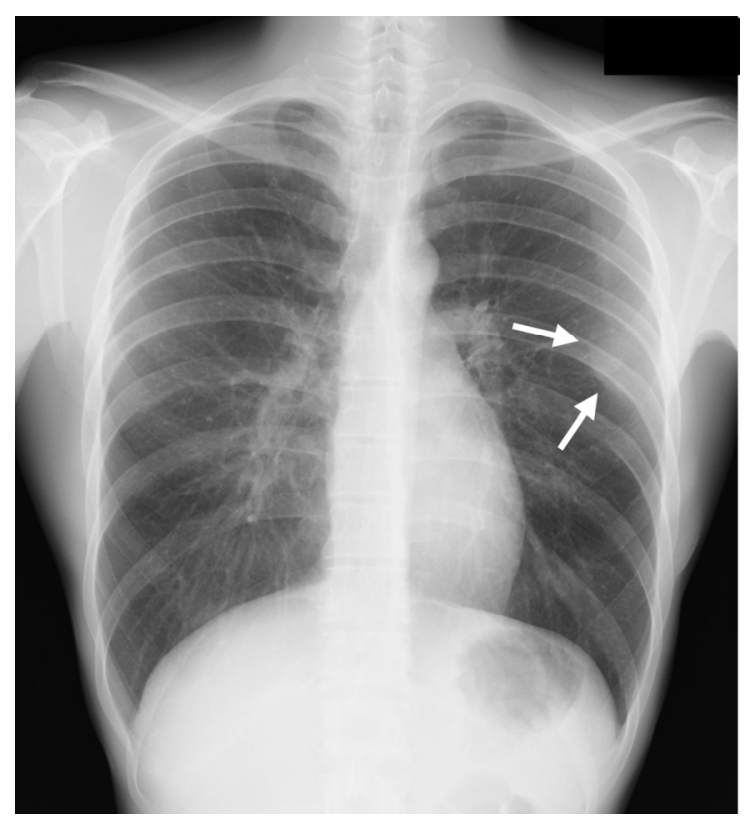

Figure 1. Chest radiography findings. Round mass in left mid lung field has incomplete border sign (arrow). 
slightly lower in the lesion than muscles and contrast-enhanced CT showed heterogeneous enhancement effect (Figure 2(b)). Magnetic resonance imaging (MRI) of the lesion showed iso signal on T1-weighted images and very high signal on T2-weighted images, respectively, compared with muscle tissue (Figures 3(a) and (b)). The signal of the lesion did not change on fat suppression images and a gadolinium-based contrast agent showed enhancement effect in the lesion (Figures 3(c) and (d)). Since these non-specific findings indicated a differential diagnosis of a neurogenic or vascular tumor, surgical excision was indicated to conclude a diagnosis based on pathological confirmation.

The cut surface of the $20 \times 10 \mathrm{~mm}$ gross reddish tumors was a glossy gray-white, and histology showed that it comprised adipocytes and blood vessels of various sizes (Figure 4(a)). The surface of the intra-thoracic side was covered with a single layer of mesothelial cells. Some vessels had thick walls surrounded by smooth muscle layers, and others had thin walls. Capillary hemangioma-like areas were evident and some small blood vessels contained microthrombi. The tumor was ill-defined and had infiltrated spaces between skeletal muscle fibers (Figure 4(b)). A focus composed of proliferative spindle cells reminiscent of angiomyolipoma was not found and a focus positive for HBM-45 was immunohistochemically undetectable. The lesion was diagnosed as infiltrating angiolipoma based on these histopathological and immunohistochemical findings.

The patient remains well without any signs of recurrence or new lesions at 1 year after surgery.

\section{DISCUSSION}

This is the first report of infiltrating angiolipoma which invades thoracic wall. Angiolipoma is a rare benign neoplasm that most frequently involves the extremities and the trunk [1,2]. Originally described by Howard and Helwig in 1960 [1], angiolipoma was classified as a non-infiltrative form and also as a rare, deeper infiltrative form involving skeletal muscle and deep soft tissues [2].

Histlogical findings of our patient show that the tumor was an infiltrating angiolipoma. Angiolipomas are predominantly composed of mature adipocytes with angiomatous proliferation. Blood vessels often contain fibrin-rich thrombi [1]. Infiltrating angiolipoma is a unencapsulated mass that tends to involve other tissues [3]. Symptoms such as muscle pain and neural deficits sometimes accompany these characteristics $[1,4]$. Surgical excision with a wide margin including surrounding normal tissue is recommended for infiltrative angiolipoma because of its aggressive behavior and the possibility of recurrence. Unlike non-infiltrative angiolipoma, this lesion is treated by local enucleation [3,5]. However, to differentiate infiltrating from non-infiltrating angiolipoma before surgi-

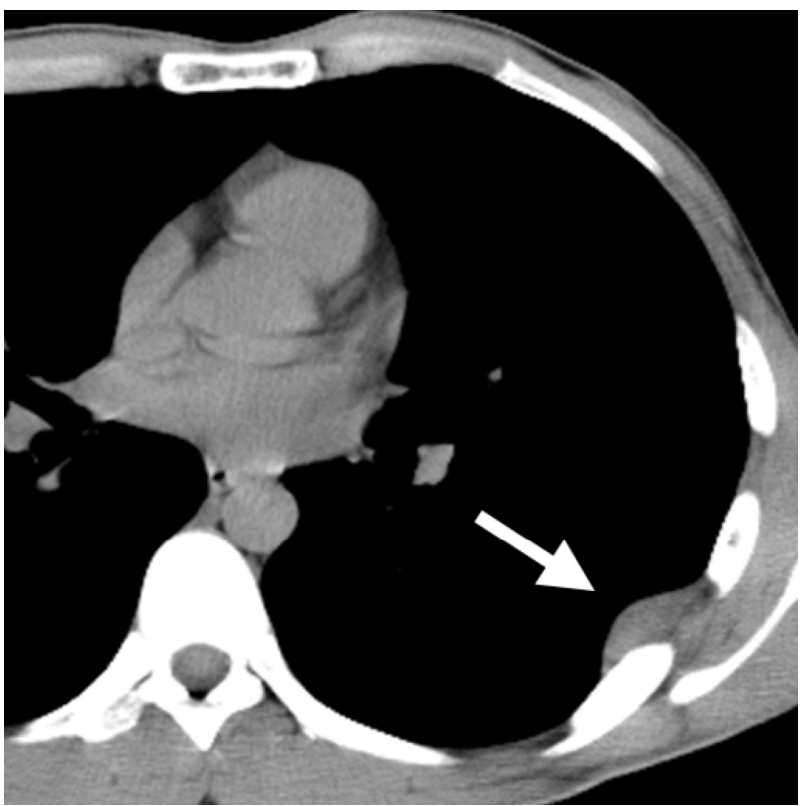

(a)

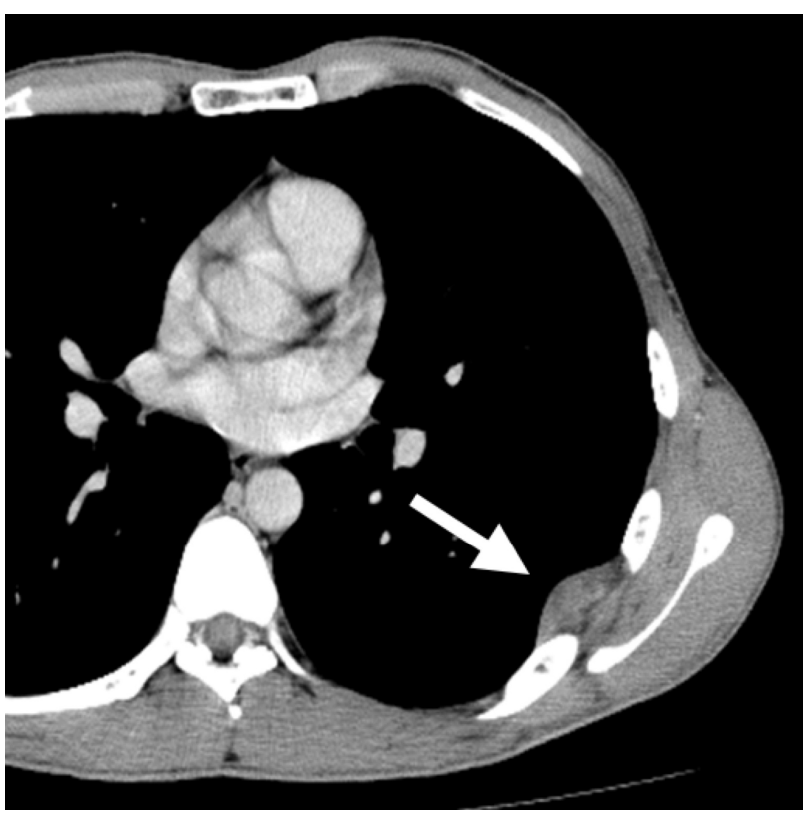

(b)

Figure 2. Chest computed tomography (CT) findings. Unenhanced CT shows dome-like mass at subpleural region of the left sixth rib and slightly lower attenuation in lesion than muscles (a); Heterogeneous contrast enhancement in lesion (b).

cal excision is difficult because imaging findings of infiltrating angiolipoma have not been described in detail.

Imaging findings can reflect the histological characteristics of infiltrating angiolipoma. One report describes infiltrating angiolipoma with serpiginous attenuation over a diffuse background of fat lucency and a poor margin on radiography [2]. Others have described infiltrating angiolipoma as being of heterogeneous density with an unclear margin on CT images suggesting various 


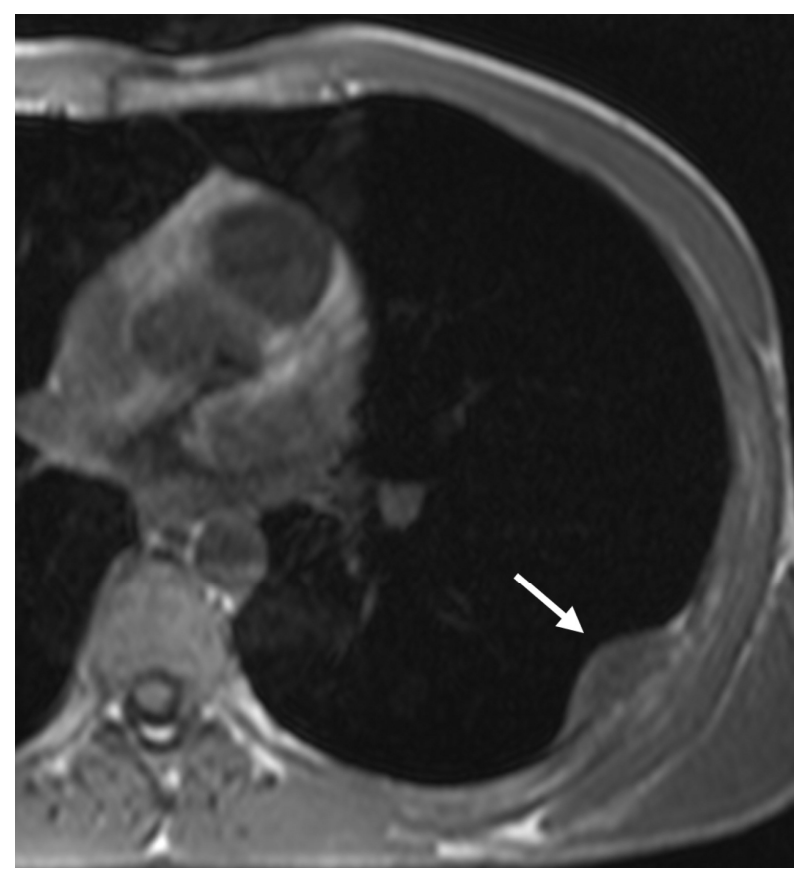

(a)

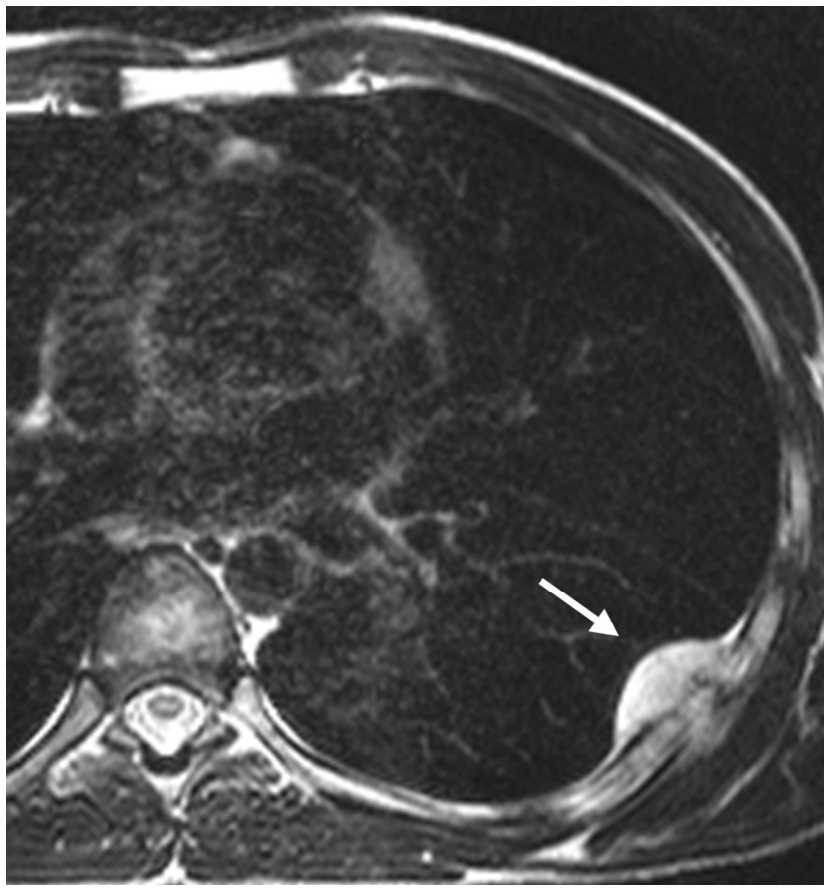

(c)

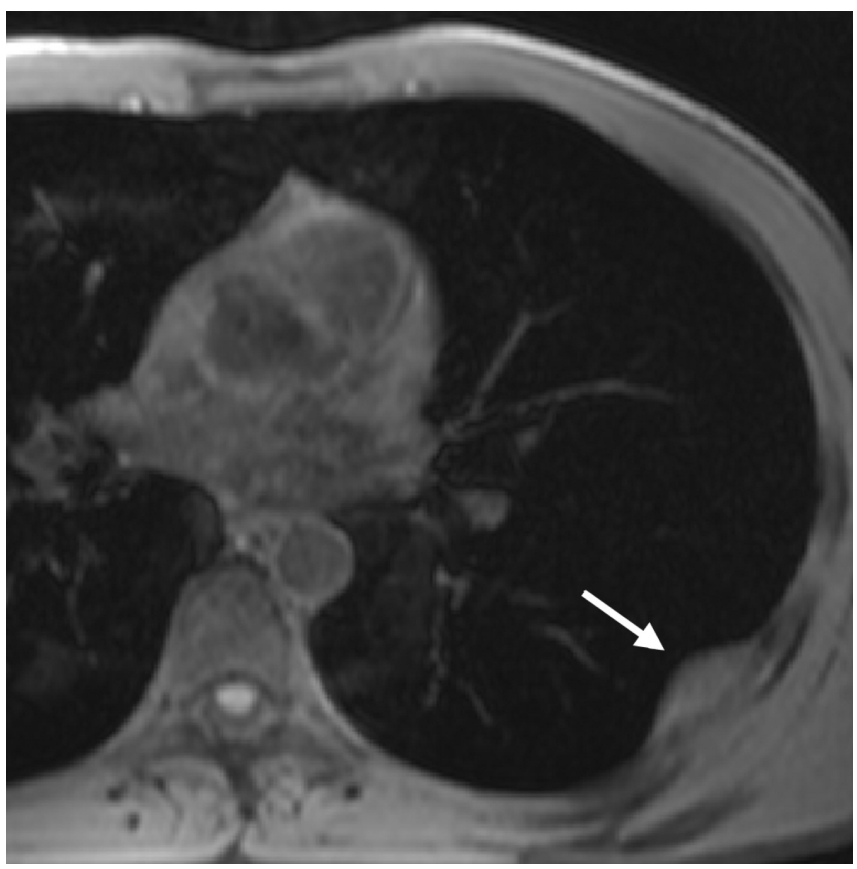

(b)

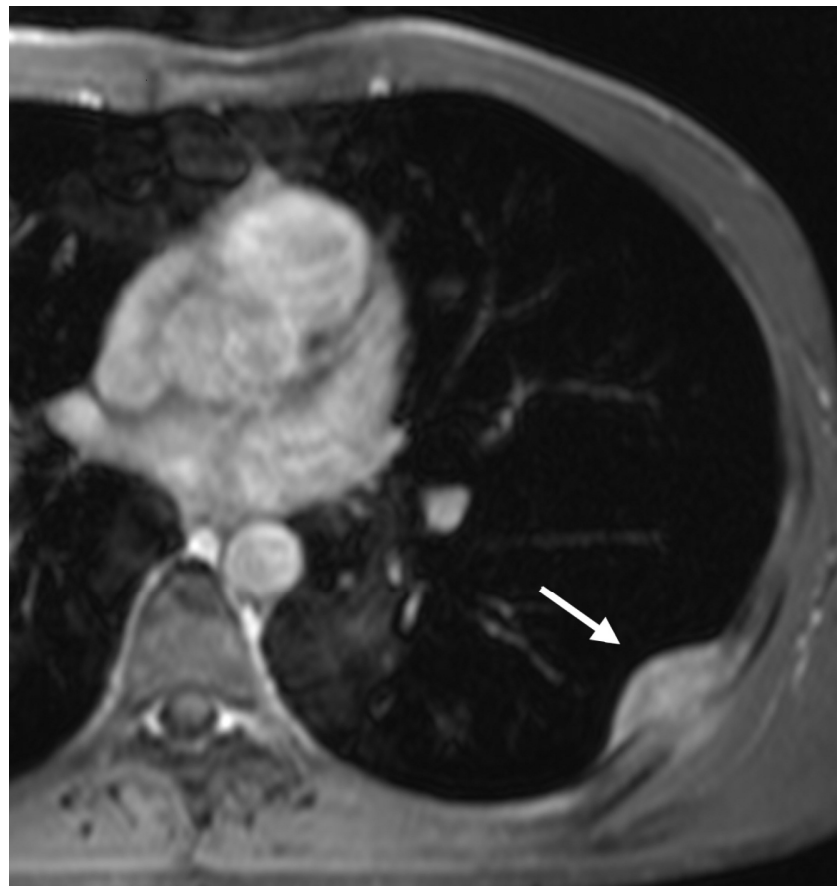

(d)

Figure 3. Magnetic resonance imaging (MRI) of the chest. Iso signal on T1-weighted (a) and fat saturated T1-weighted (b) images. High signal on T2-weighted image (c). Contrast enhancement effect in the lesion (d).

components such as adipose tissues, vascular components and muscle, and having a central area of low attenuation surrounded by areas of contrast enhancement on enhanced CT images [4]. These reports indicate that a central area of low attenuation reflects a lipomatous component, whereas contrast enhancement in other areas reflects a vascular component [4]. Findings of MRI might support these notions, as such tumors almost always comprise two components. Homogeneous high signal on both T1- and T2-weighted images with decreased signal on fat suppression images reflects a lipomatous component, whereas heterogeneous high signal on T2-weighted images reflects a vascular component $[4,6]$. In addition to these findings and dependently on 


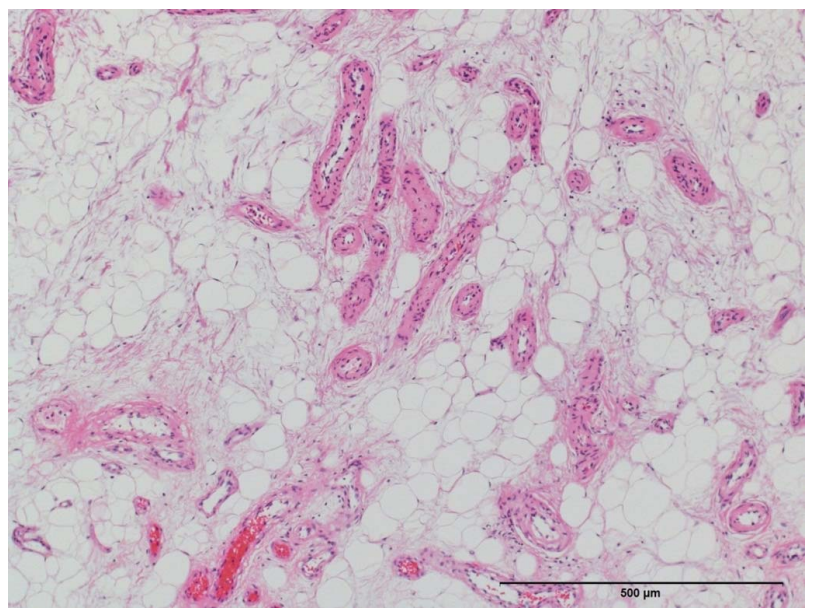

(a)

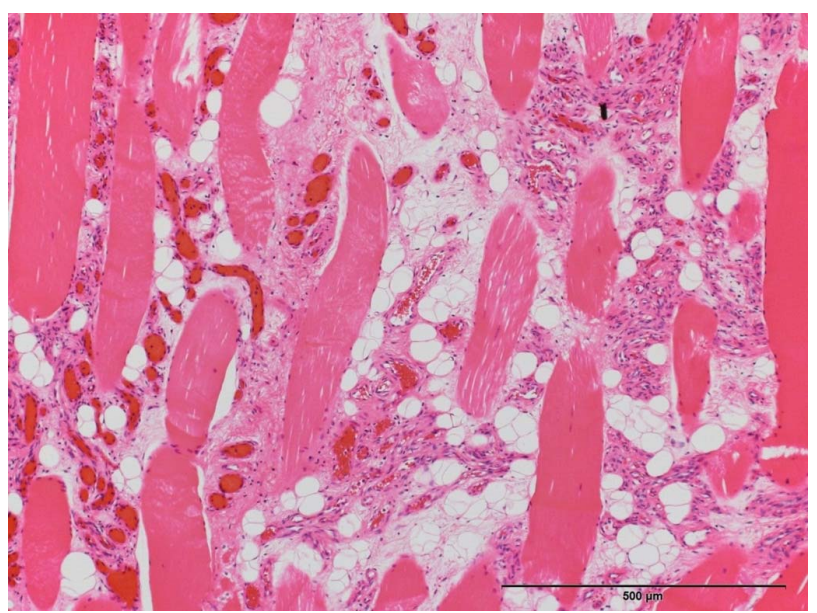

(b)

Figure 4. High-power view of tumor $(\times 100$, H-E stain $)$. Adipocytes and blood vessels of various sizes (a); Ill-defined lesion has infiltrated skeletal muscle fibers (b).

the internal components of a lesion, invasion within surrounding structures is clearer on MRI than CT images.

In this case, it was difficult to diagnosis the tumor as angiolipoma before excision because no fat component was revealed in the tumor on MRI. We speculate that adipose and vascular component was admixed in a same voxel so that it was difficult to suppress fat signal using chemical shift selective (CHESS) technique. Chemical shift imaging using gradient echo sequence imaging might have revealed a signal change [7].

In conclusion, infiltrating angiolipoma can be included in a differential diagnosis of thoracic wall lesions that show high intensity signal on T2-weighted images and a contrast enhancement effect, although infiltrating angiolipoma is an extremely rare neoplasm. This tumor was difficult to preoperatively diagnose in our patient because of the unusual location and atypical imaging findings.

\section{REFERENCES}

[1] Howard, W.R. and Helwig, E.B. (1960) Angiolipoma. Archives of Dermatology, 82, 924-931. doi:10.1001/archderm.1960.01580060078011

[2] Gonzalez-Crussi, F., Enneking, W.F. and Arean, V.M. (1966) Infiltrating angiolipoma. Journal of Bone and Joint Surgery American, 48, 1111-1124.

[3] Lin, J.J. and Lin, F. (1974) Two entities in angiolipoma. A study of 459 cases of lipoma with review of literature on infiltrating angiolipoma. Cancer, 34, 720-727. doi:10.1002/1097-0142(197409)34:3<720::AID-CNCR2 820340331>3.0.CO;2-K

[4] Arenaz, B.J., Luaces, R., Lorenzo, F.F., Garcia-Rozado, A., Crespo, E.J.L., Fronseca, C.E. and Lopez-Cedrun, J.L. (2010) Angiolipoma in head and neck: Report of two cases and review of the literature. International Journal of Oral and Maxillofacial Surgery, 39, 610-615. doi:10.1016/j.ijom.2009.12.021

[5] Murphey, M.D., Carroll, J.F., Flemming, D.J., Pope, T.L., Gannon, F.H. and Kransdorf, M.J. (2004) From the archives of the AFIP: Benign musculoskeletal lipomatous lesions. Radiographics, 24, 1433-1466. doi:10.1148/rg.245045120

[6] Matsuoka, Y., Kurose, K., Nakagawa, O. and Katsuyama, J. (1988) Magnetic resonance imaging of infiltrating angiolipoma of the neck. Surgical Neurology, 29, 62-66. doi:10.1016/0090-3019(88)90124-3

[7] Hochhegger, B., Marchiori, E., dos Reis, D.Q., Souza Jr., A.S., Souza, L.S., Brum, T. and Irion, K.L. (2012) Chemical-shift MRI of pulmonary hamartomas: Initial experience using a modified technique to assess nodule fat. American Journal of Roentgenology, 199, W331-W334. doi:10.2214/AJR.11.8056 